\title{
Primary Hyperparathyroidism in Pregnancy: A Case Report
}

\author{
Cristina Zanardini ${ }^{\mathrm{a}}$, Rossana Orabona ${ }^{\mathrm{a}, \mathrm{b}, \mathrm{f}}$, Sonia Zatti ${ }^{\mathrm{a}}$, Carlo Cappellic, \\ Claudio Casella ${ }^{\mathrm{d}}$, Carla Baronchellie, Enrico Sartori ${ }^{\mathrm{a}, \mathrm{b}}$
}

\begin{abstract}
Primary hyperparathyroidism (PHP) is the third most common endocrine disorder. We report the case of a 28 -year-old woman who experienced general weakness, hyperemesis gravidarum and hypercalcemia at 11 weeks of gestation. Corresponding to hypercalcemia, we found inappropriately elevated parathyroid hormone levels. Through neck computed tomography a solitary adenoma of the parathyroid gland, measuring $6 \times 2.9 \times 11 \mathrm{~mm}$ has been documented. An ultrasound-guided fine needle aspiration from the mass confirmed the suspicious of a benign tumor. Left superior parathyroidectomy resulted in immediate and permanent resolution of hypercalcemia. The postoperative course was uneventful. Histopathological and immunohistochemical analyses were consistent with parathyroid adenoma. The diagnostic approach to hypercalcemia in pregnancy represents a challenge for multidisciplinary teamwork.
\end{abstract}

Keywords: Neuroendocrine tumor; Primary hyperparathyroidism; Hypercalcemia; Parathyroidectomy; Pregnancy

\section{Introduction}

Endocrine disorders in pregnancy are challenging as regards diagnosis and treatment. Primary hyperparathyroidism (PHP) is the third most common endocrine disorder, often asymptomatic. We report the management of a symptomatic case during pregnancy.

\section{Case Report}

In April 2019 (April 27), a 28-year-old woman gravida 3/para

Manuscript submitted March 12, 2020, accepted March 23, 2020

a Obstetrics and Gynecology, ASST Spedali Civili, Brescia, Italy

bDepartment of Clinical and Experimental Sciences, University of Brescia, Brescia, Italy

'Department of Clinical and Experimental Sciences, Endocrine and Metabolic Unit, Medical Clinic, University of Brescia, Brescia, Italy

${ }^{\mathrm{d} D e p a r t m e n t}$ of Molecular and Translational Medicine, ASST Spedali Civili, 3rd Division of General Surgery, University of Brescia, Brescia, Italy

eInstitute of Pathology, ASST Spedali Civili, Brescia, Italy

${ }^{\mathrm{f}}$ Corresponding Author: Rossana Orabona, Piazzale Spedali Civili 1, Brescia

25123, Italy. Email: rossana.orabona@gmail.com

doi: https://doi.org/10.14740/jmc3441
1 at 11 weeks of gestation with no previous known diseases, presented to the Obstetrics Department of the University Hospital of Brescia, Italy, with symptoms of general weakness, hyperemesis gravidarum and hypercalcemia. There was no family history of parathyroid disease, hypercalcemia, nephrolithiasis, or other endocrinopathies. At admission the physical examination revealed tachycardia, and serum calcium was 12.2 $\mathrm{mg} / \mathrm{dL}$. Thyroid ultrasound revealed an heterogeneous mass along the inferior margin of the left thyroid gland, measuring $8 \mathrm{~mm}$. Laboratory investigations included total calcium level $11.95 \mathrm{mg} / \mathrm{dL}$ (normal range, 8.10 - 9.80), parathyroid hormone (PTH) $142 \mathrm{pg} / \mathrm{mL}$ (normal range, 11 - 67), phosphorus $1.8 \mathrm{mg} /$ $\mathrm{dL}$ (normal range, 2.5 - 4.3), serum albumin $4.02 \mathrm{~g} / \mathrm{dL}$ (normal range, 3.40 - 4.65), thyroid stimulating hormone (TSH) 0.083 $\mathrm{mIU} / \mathrm{L}$ (normal range, $0.270-4.200$ ) and fT4 22.80 (normal range, 9.30 - 17.00). Biochemical hyperthyroidism was interpreted as consistent with pregnancy-induced rise of beta human chorionic gonadotropin. A fine needle aspiration from the mass demonstrated scant cells, and it was reported as benign cytology. She was diagnosed with PHP. Conservative treatment with intravenous fluid (lactated ringers $500 \mathrm{~mL} /$ day and normal saline $1,000 \mathrm{~mL} /$ day) and furosemide $20 \mathrm{mg} /$ die was started, monitoring blood pressure, daily diuresis and body weight. Meanwhile obstetric evaluation showed appropriate for gestational age fetal biometry. Neck computed tomography 4D showed a solitary adenoma of the parathyroid gland, measuring $6 \times 2.9 \times 11 \mathrm{~mm}$ (Fig. 1).

On May 15 (14 weeks of gestation), the patient underwent cervical exploration with left superior parathyroidectomy. The histopathology showed a benign adenoma (Fig. 2). The basal level of PTH was $151 \mathrm{pg} / \mathrm{mL}$, within 10 min after the parathyroid adenoma was removed the PTH level decreased to $12 \mathrm{pg} /$ $\mathrm{mL}$. Intravenous glucose solution $5 \%(500 \mathrm{~mL})$ with calcium gluconate $10 \%$ was started in the first $36 \mathrm{~h}$ after surgery and then was continued with oral calcium $1,000 \mathrm{mg}$ two times a day. Total calcium level at the end of the intravenous calcium infusion was $8.88 \mathrm{mg} / \mathrm{dL}$. Vitamin D supplementation $(25,000$ $\mathrm{IU} /$ week) for 6 weeks (and then one time a month) was also started and continued for 6 weeks. The postoperative period was uneventful; in particular she did not develop hypocalcemia after surgery. Her serum calcium and PTH levels normalized; on May 22, PTH was $31 \mathrm{pg} / \mathrm{mL}$ and total calcium level $8.99 \mathrm{mg} / \mathrm{dL}$. She was discharged on May 30.

In subsequent weekly follow-up, her serum calcium and PTH levels have been within the normal limits. On October 30 , at 38 weeks of gestation, the patient gave birth to a healthy female baby of adequate weight. Ethical approval was not required based on national laws regarding anonymized data. 


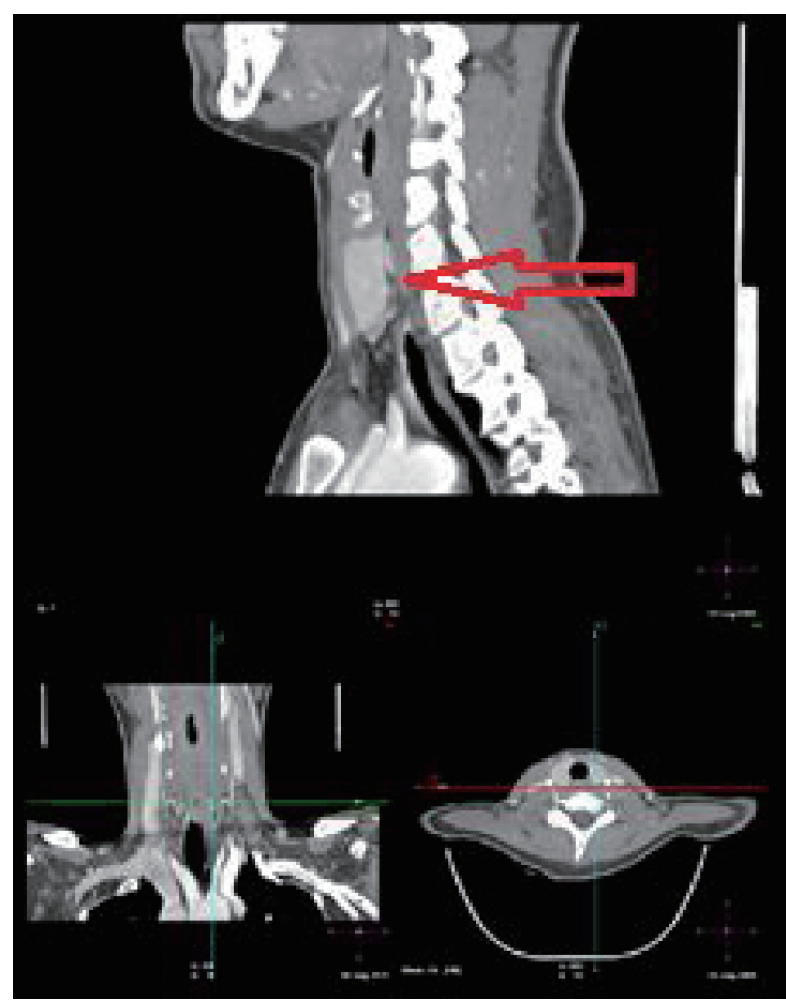

Figure 1. Computed tomography (CT) images of parathyroid adenoma (arrow).

\section{Discussion}

Worldwide, PHP (overproduction of PTH due to parathyroid hyperplasia, adenoma or carcinoma) is the third most common endocrine disorder, after diabetes and thyroid disease; and women are twice as likely to be affected as men [1].
Symptoms of hypercalcemia occur in $20 \%$ of patients and include fatigue, nausea, constipation, depression, renal impairment and cardiac arrhythmias. Its occurrence during pregnancy is rare with a reported incidence of $1 \%$, usually due to a parathyroid adenoma, which is present in approximately $80 \%$ of all cases, and often remains undiagnosed due to physiological changes during pregnancy that mask the symptoms (e.g. hypoalbuminemia, calcium transport across the placenta, increased glomerular filtration rate, estrogenic inhibition of PTH-mediated bone resorption). It is considered that as many as $80 \%$ of cases of PHP during pregnancy may be asymptomatic. However, untreated PHP can be associated with serious maternal (e.g. hypercalcemic crisis, pancreatitis, nephrolithiasis, preeclampsia) and fetal (e.g. neonatal tetany, hypoparathyroidism, stillbirth, intrauterine growth restriction, preterm delivery, and miscarriage) complications which may be as high as $67 \%$ and $80 \%$ respectively, directly proportionate to degree of maternal serum calcium levels. Because neonatal mortality is associated with maternal hyperparathyroidism, early diagnosis is essential.

The optimal management should be individualized according to the clinical course, gestational age, the severity of hypercalcemia and the risk for each treatment option for the mother and the baby. In asymptomatic patients with mild hypercalcemia $(<11 \mathrm{mg} / \mathrm{dL})$, conservative treatment with oral hydration and low-calcium diet, with or without forced diuresis with a low dose of loop diuretic can produce good outcomes for both the mother and the baby [2]. Most clinical studies and guidelines recommend parathyroidectomy as the treatment of choice for PHP occurring in pregnancy, and it seems to be the safest beyond the second trimester $[3,4]$.

\section{Acknowledgments}

None to declare.

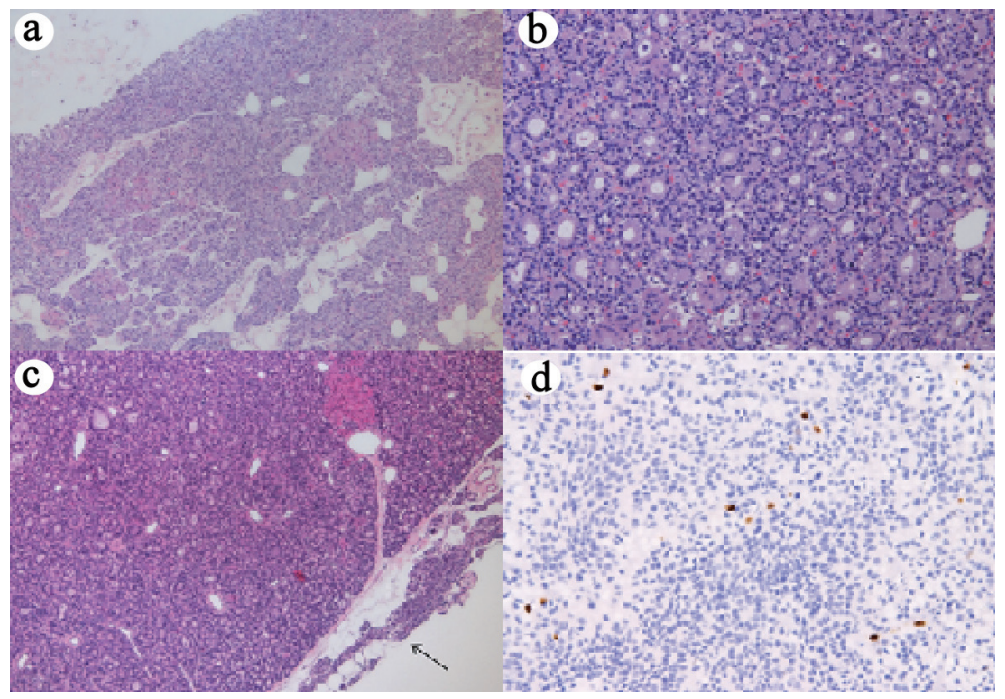

Figure 2. (a) The microtubular and trabecular architecture of the adenoma $(H \& E, \times 4)$. (b) Adenoma: complete disappearance of adipose tissue inside the parenchyma (H\&E, $\times 10)$. (c) Normal parathyroid parenchyma (black arrow) at the peripheral side of the lesion (H\&E, × 4). (d) Positive immunohistochemistry for Ki67 (× 10), <1\%. H\&E: hematoxylin and eosin stain. 


\section{Financial Disclosure}

None to declare.

\section{Conflict of Interest}

All authors have nothing to declare.

\section{Informed Consent}

The patient gave her informed consent.

\section{Author Contributions}

CZ and RO wrote the paper. SZ, C. Cappelli, C. Casella, CB and $\mathrm{ES}$ revised the paper as regards their specialty.

\section{Data Availability}

Any inquiries regarding supporting data availability of this study should be directed to the corresponding author.

\section{References}

1. Yeh MW, Ituarte PH, Zhou HC, Nishimoto S, Liu IL, Harari A, Haigh PI, et al. Incidence and prevalence of primary hyperparathyroidism in a racially mixed population. J Clin Endocrinol Metab. 2013;98(3):1122-1129.

2. Diaz-Soto G, Linglart A, Senat MV, Kamenicky P, Chanson P. Primary hyperparathyroidism in pregnancy. Endocrine. 2013;44(3):591-597.

3. Schnatz PF, Curry SL. Primary hyperparathyroidism in pregnancy: evidence-based management. Obstet Gynecol Surv. 2002;57(6):365-376.

4. Kelly TR. Primary hyperparathyroidism during pregnancy. Surgery. 1991;110(6):1028-1033; discussion 1033-1024. 